\title{
Health eRide: Outcomes of a Pilot Program Leveraging Principles of Gamification and SMS Messaging to Help Veterans Self-Manage Chronic Pain
}

Sara Johnson, PhD; Deborah Levesque, PhD; Lynne Broderick, MPH

Pro-Change Behavior Systems, Inc, South Kingstown, RI, United States

\section{Corresponding Author:}

Lynne Broderick, MPH

Pro-Change Behavior Systems, Inc

1174 Kingstown Road

Suite 101

South Kingstown, RI, 02879

United States

Phone: 14013602978

Fax: 14013602978

Email: 1broderick@prochange.com

\section{Abstract}

Background: Chronic pain creates a significant public health burden and disproportionately affects veterans. Over $56 \%$ of Operation Iraqi Freedom (OIF), Operation Enduring Freedom (OEF), and Operation New Dawn (OND) veterans have a diagnosis of chronic pain. The frequency and extent of pain, posttraumatic stress disorder (PTSD), traumatic brain injury, and the co-occurrence of all three conditions (post-deployment multi-symptom disorder, PMD) can complicate and reduce the effectiveness of traditional treatments for pain. Taken in combination with the opioid crisis in the United States, there is an urgent need for innovative and integrative approaches to non-medical pain management. However, readiness to engage in pain self-management varies.

Objective: The objective of this Phase I pilot was to design, develop, and establish the feasibility of a theoretically-grounded, mobile-optimized, interactive pain self-management intervention for veterans that leverages principles of gamification.

Methods: The development of Health eRide: Your Journey to Managing Pain included extensive end-user input including a veteran advisory panel $(n=5)$, formative focus groups $(n=20)$, and iterative usability testing $(n=20)$. Health eRide is tailored not only to veterans' preference for pain self-management strategies but also their readiness to adopt those strategies as measured by a Transtheoretical Model-based stage of change assessment. The program incorporates core components of promising treatments for PMD by intervening on sleep hygiene and stress management. Health eRide includes (1) an online computer-tailored intervention that provides tailored behavior change guidance and (2) an individually tailored subway-themed "map" of their pain management journey. Stops along the map include stage-based interactive activities designed to further users along in their journey to self-managing pain. The program also included optional text messaging and a Facebook page. A pilot study with a 30-day follow-up was conducted with 69 veterans (81\% male, 58\% Caucasian, average age=50) to examine the acceptability and preliminary effectiveness of Health eRide. Users completed stage of change algorithms and pain rating scales at baseline and follow-up and completed a measure of acceptability and the System Usability Scale (SUS) at follow-up.

Results: A total of 44 veterans (64\%) completed the follow-up assessment (no significant differences on baseline demographics between those who completed the follow-up assessment and those who did not). The mean rating of acceptability for Health eRide was 3.20 on a 4 point scale, indicating that users found the program useful, informative, reliable, and easy to navigate. Likewise, the mean score on the SUS was 65.4, indicating the program meets standards for usability. The results show significant changes in movement to readiness to adopt strategies to self-manage pain (54.5\% at baseline vs $79.5 \%$ at follow-up, $P=.007)$ and effectively manage stress $(50 \%$ vs $88.6 \%, P<.001)$. While there was movement to readiness to adopt healthy sleep habits $(25 \%$ vs $38.6 \%$ ), the change was not significant. There were significant improvements in ratings of pain: "How would you rate your pain right now?" (partial $\eta 2=.205, P=.002$ ) and "How would you rate your usual pain in the last week?" (partial $\eta 2=.378, P<.001$ ).

Conclusions: Findings provide encouraging evidence of the acceptability and effectiveness of incorporating principles of gamification into a theoretically grounded intervention for veterans at all levels of readiness for self-managing chronic pain. 
(iproc 2016;2(1):e29) doi: 10.2196/iproc.6155

\section{KEYWORDS}

gamification; intervention; veterans; serious games; pain management

This poster was presented at the Connected Health Symposium is displayed as an image in Figure 1 and as a PDF in Multimedia 2016, October 20-21, Boston, MA, United States. The poster Appendix 1.

Figure 1. Poster.

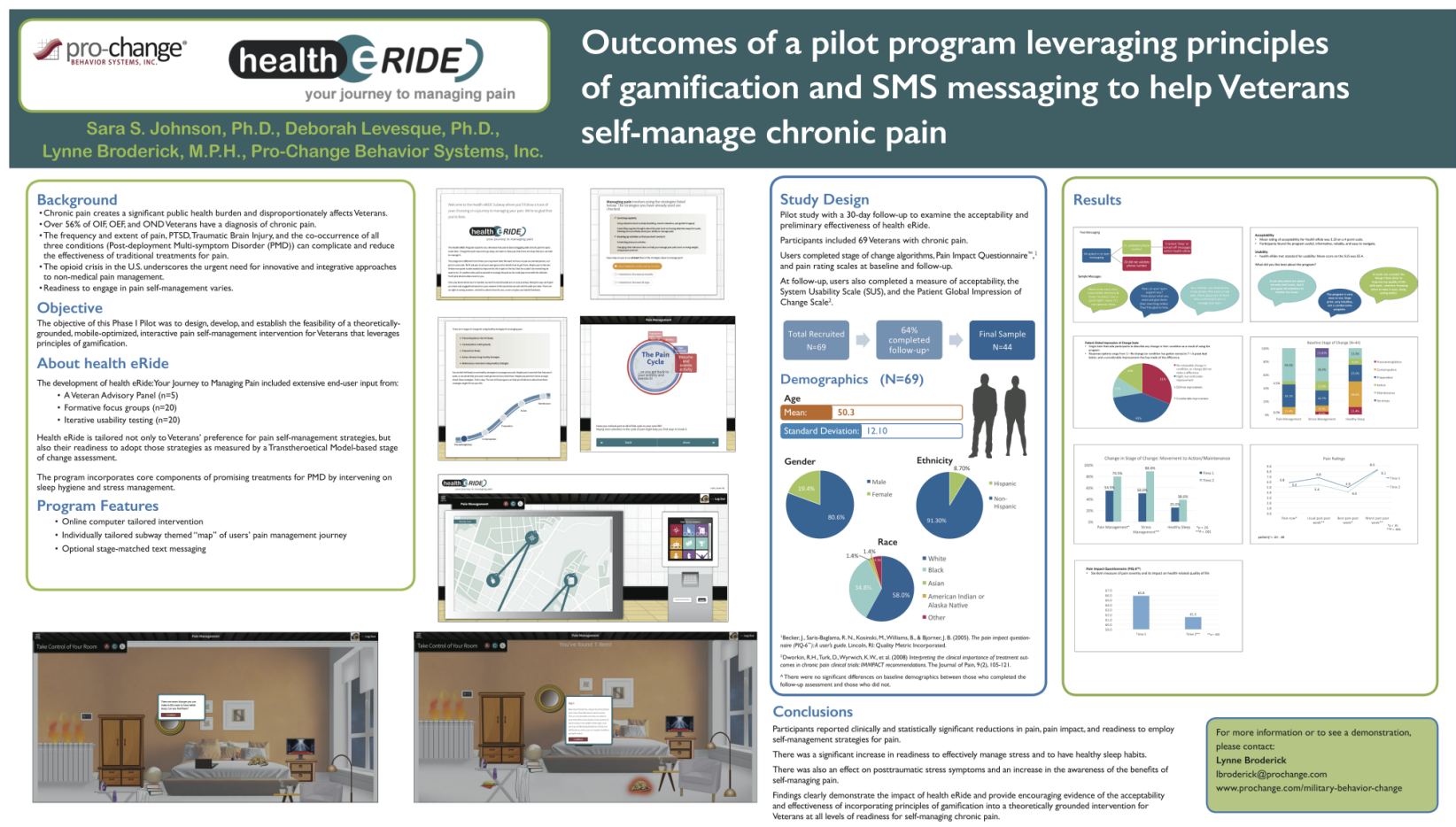

\section{Acknowledgments}

This research was supported by the National Center for Advancing Translational Sciences (NCATS), Grant \#1R43TR000362-01A1.

\section{Multimedia Appendix 1}

Poster.

[PDF File (Adobe PDF File), 5MB-Multimedia Appendix 1]

Edited by T Hale; submitted 05.06.16; peer-reviewed by CHS Scientific Program Committee; accepted 02.08.16; published 29.12.16
Please cite as:
Johnson S, Levesque D, Broderick L
Health eRide: Outcomes of a Pilot Program Leveraging Principles of Gamification and SMS Messaging to Help Veterans Self-Manage
Chronic Pain
iproc $2016 ; 2(1): e 29$
URL: $\underline{\text { http://www.iproc.org/2016/1/e29/ }}$
doi: $10.2196 /$ iproc.6155
PMID:

CSara Johnson, Deborah Levesque, Lynne Broderick. Originally published in Iproceedings (http://www.iproc.org), 29.12.2016. This is an open-access article distributed under the terms of the Creative Commons Attribution License (http://creativecommons.org/licenses/by/2.0/), which permits unrestricted use, distribution, and reproduction in any medium, 
provided the original work, first published in Iproceedings, is properly cited. The complete bibliographic information, a link to the original publication on http://www.iproc.org/, as well as this copyright and license information must be included. 